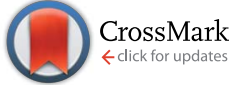

Cite this: RSC Adv., 2017, 7, 4253
Received 15th October 2016 Accepted 15th December 2016

DOI: 10.1039/c6ra25294j

www.rsc.org/advances

\section{VEGF-loaded biomimetic scaffolds: a promising approach to improve angiogenesis and osteogenesis in an ischemic environment}

\author{
Bo Li, ${ }^{a}$ Hai Wang, ${ }^{a}$ Gang Zhou, ${ }^{b}$ Jing Zhang, ${ }^{b}$ Xinlin Su, ${ }^{c}$ Zhifeng Huang, ${ }^{a}$ Qiang Li, ${ }^{a}$ \\ Zhihong $W^{* a d e}$ and Guixing Qiu*a
}

The treatment of bone defects in an ischemic environment is a huge challenge. Improving angiogenesis, which is regulated by angiogenic growth factors such as vascular endothelial growth factor (VEGF), may have the potential to enhance bone regeneration. This study was conducted to investigate whether VEGF-loaded biomimetic scaffolds could improve angiogenesis and osteogenesis in an ischemic limb. The biomimetic hydroxyapatite-collagen scaffold (HC scaffold) was fabricated by adopting the freeze-drying approach. In the HC-VEGF group, VEGF was incorporated into $\mathrm{HC}$ scaffolds. The cytocompatibility of the HC scaffold was evaluated by CCK- 8 testing. The release profile of VEGF from the HC-VEGF scaffold was detected by using VEGF ELISA kits. The femoral artery of Sprague-Dawley rats was resected to induce an ischemic environment. HC scaffolds with or without VEGF were implanted into intramuscular pockets in the ischemic limb. Eight weeks after implantation, samples were retrieved for histological and immunohistochemical analysis. The HC scaffolds showed good cytocompatibility according to the results of a CCK- 8 test. In vitro, an initial burst release ( $80 \%)$ of VEGF was detected during the first 3 days. Angiogenesis and osteogenesis were significantly enhanced in the HC-VEGF group, compared to the control group of HC scaffolds without VEGF. This study provided a very promising approach to enhance angiogenesis and osteogenesis in an ischemic environment by incorporating VEGF into biomimetic bone scaffolds.

\section{Introduction}

Bone tissue is highly vascularized from the marrow cavity to the periosteum. ${ }^{\mathbf{1}, 2}$ Since oxygen diffusion is limited to $200 \mu \mathrm{m}$ from the nearest capillary, successful bone regeneration relies on the

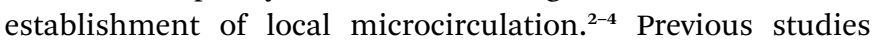
have illuminated that the modulation of angiogenesis could markedly influence bone formation. ${ }^{5-7}$ Inhibiting angiogenesis is detrimental to bone regeneration ${ }^{5,8}$ and improving

\footnotetext{
${ }^{a}$ Department of Orthopaedic Surgery, Peking Union Medical College Hospital, Peking Union Medical College and Chinese Academy of Medical Sciences, No. 1 Shuaifuyuan, Beijing 100730, China.E-mail: qiugx@medmail.com.cn;wuzh3000@ 126.com; Tel: +86-10-6915-2800; +86-10-6915-2807

${ }^{b}$ Key Laboratory for Biomechanics and Mechanobiology of Ministry of Education, School of Biological Science and Medical Engineering, Beihang University, Beijing 100191, China

'Department of Orthopaedic Surgery, The First Affiliated Hospital of Soochow University, Suzhou, Jiangsu, China

${ }^{d}$ Central Laboratory, Peking Union Medical College Hospital, Peking Union Medical College and Chinese Academy of Medical Sciences, No. 1 Shuaifuyuan, Beijing 100730, China

${ }^{e}$ Beijing Key Laboratory for Genetic Research of Bone and Joint Disease, No. 1 Shuaifuyuan, Beijing 100730, China
}

vascularization is beneficial for bone formation. ${ }^{5,6}$ Currently, it is realized that the preservation and reconstruction of blood supply is a key factor in fracture healing.

Unfortunately, in some pathological conditions, such as trauma, diabetes and arterial occlusion, blood perfusion is severely impaired in the affected limbs. ${ }^{9-11}$ Inadequate blood supply is a huge challenge for the successful treatment of bone fractures or defects., ${ }^{\mathbf{9 2 , 1 3}}$ It was reported that the delayed- and non-union rate were three times higher in patients with concomitant arteries disruption than those without vascular injury. ${ }^{13}$ An in vivo study by Lu et al. indicated that ischemic insult could lead to a delayed- or non-union. ${ }^{9}$ Thus, exploring effective approach to enhance bone regeneration in an ischemic environment is of great significance, and stimulating angiogenesis may have the potential to improve bone formation in this condition. A variety of strategies have been developed to improve vascularization, which is called therapeutic angiogenesis, in peripheral ischemic diseases. ${ }^{14-16}$ The delivery of angiogenic growth factors is a classical approach, since vascularization is regulated by these cytokines.,17 Among them, vascular endothelial growth factor (VEGF) is a key mediator in angiogenesis. It has been proven to be effective to increase blood perfusion and stimulate angiogenesis in skeletal muscle 
tissues of the ischemic limb. ${ }^{\mathbf{1 5}, 16}$ However, whether VEGF released from biomimetic bone scaffolds could improve angiogenesis and osteogenesis in an ischemic limb is still unknown.

In this study VEGF was incorporated into biomimetic hydroxyapatite-collagen scaffolds (HC scaffolds) to investigate its ability to enhance angiogenesis and osteogenesis in an ischemic environment. HC scaffold was adopted due to its biodegradable, biocompatible, osteoconductive and osteoinductive properties. ${ }^{18-20}$ An ischemic model of femoral artery resection was used in this study, which has been demonstrated to be able to significantly reduce blood supply in affected limbs., ${ }^{\mathbf{9 1 2 , 2 1 , 2 2}}$ Hypothesis of this study is that the release of VEGF from HC scaffold can enhance angiogenesis and osteogenesis in an ischemic environment.

\section{Experimental}

\subsection{Scaffold fabrication}

Collagen was extracted from bovine tendon and prepared using an enzyme digestion method. Briefly, bovine tendon was cleaned of fascia and muscle tissues and freeze-dried for $48 \mathrm{~h}$. The dried bovine tendon was cut into small pieces and treated with distilled water using the method of homogenate extraction for 2-3 times, followed by centrifugation at $5000 \mathrm{rpm}$ for $15 \mathrm{~min}$. The precipitation was suspended in $1.5 \mathrm{~L}$ acetic acid solution $(0.5 \mathrm{M})$ containing $2 \mathrm{mg}$ trypsin (Sigma, USA) and incubated at $4{ }^{\circ} \mathrm{C}$ for $24 \mathrm{~h}$. Sodium chloride $(2.4 \mathrm{~mol}$ $\mathrm{L}^{-1}$ ) was added to the mixed solution, followed by centrifugation at $5000 \mathrm{rpm}$ for $15 \mathrm{~min}$. The precipitation was redissolved in $0.5 \mathrm{M}$ acetic acid. The above steps were repeated 3 times for purifying collagen. Collagen acetic acid solution was then dialyzed for $72 \mathrm{~h}$ using dialysis tube (molecular weight cut off $=30 \mathrm{kDa}$ ) (Sigma). Disodium hydrogen phosphate solution $\left(\mathrm{Na}_{2} \mathrm{HPO}_{4} ; 0.2 \mathrm{M}\right)$ and phosphate buffer saline (PBS; $1 \times$ ) were selected as dialysis buffer. Dialyzed collagen was freeze-dried for $48 \mathrm{~h}$ and saved in $4{ }^{\circ} \mathrm{C}$ freezer for the following experiment.

The hydroxyapatite-collagen scaffold was fabricated by adopting the freeze-drying approach. The extracted collagen was swollen in $0.5 \mathrm{M}$ acetic acid to a concentration of $40 \mathrm{mg}$ $\mathrm{mL}^{-1}$, followed by constant stirring for $24 \mathrm{~h}$ with a homogenizer. After the collagen was mixed thoroughly, hydroxyapatite powder (Shanghai Jinsui Biotechnology Co., Ltd., Shanghai, China) was added into slurry with constant stirring for 24 hours. During the process of stirring, 1-ethyl-3-[3dimethylaminopropyl] carbodiimide hydrochloride (EDC)/ $N$-hydroxysuccinimide (NHS) $(50 \mathrm{mM}, 50 \mathrm{mM})$ were added to the slurry as crosslink agent. VEGF $(4 \mu \mathrm{g}$, Beijing Wishbiotechnology Co., Ltd., Beijing, China) was added in the composite scaffold group (HC-VEGF scaffold group). The final ratio of HA was adjusted to $70 \%$. Then, the slurry was injected into the cylindrical molds with diameter of $4 \mathrm{~mm}$ and length of $15 \mathrm{~mm}(\varnothing 4 \times 15)$ after degassing treatment. A constant pressure of $10 \mathrm{MPa}$ for 2 hours was applied on the material to improve its mechanical strength. Finally, materials were refrigerated at $-70{ }^{\circ} \mathrm{C}$ overnight, and lyophilized at
$-40{ }^{\circ} \mathrm{C}$ for $48 \mathrm{~h}$. Cylindrical $\mathrm{HC}$ and HC-VEGF scaffold (diameter: $4 \mathrm{~mm}$ ) was cut into $5 \mathrm{~mm}$ in height for further experimental assessment. The morphology and microstructures of scaffolds were evaluated by scanning electron microscopy (SEM, S-2400; Hitachi, Japan), after sputtercoated with gold.

\subsection{Preparation of scaffold extracts}

HC scaffolds were sterilized under UV light for $2 \mathrm{~h}$ and washed with sterile PBS three times. Scaffold extracts were prepared according to a standard procedure (ISO 10993-5). Briefly, HC scaffolds were immersed in $\alpha$-MEM medium (Gibco, Grand Island, NY, USA) containing 10\% fetal bovine serum (FBS, Invitrogen, Carlsbad, USA) and $1 \%$ penicillin/streptomycin at $37^{\circ} \mathrm{C}$ for $24 \mathrm{~h}$. The supernatant was collected and sterilized by filtering with a $0.22 \mu \mathrm{m}$ filter for further evaluating the cytocompatibility of scaffolds.

\subsection{Cell viability}

MC3T3-E1 cells (Institute of Basic Medical Sciences, Peking Union Medical College and Chinese Academy of Medical Sciences, Beijing, China) were seeded in a 96-well plate (5000 cells per well) and cultured in $\alpha$-MEM medium supplemented with $10 \%$ fetal bovine serum and $1 \%$ penicillin/streptomycin under $5 \% \mathrm{CO}_{2}$ at $37{ }^{\circ} \mathrm{C}$. Twenty four hours later, cells were treated with HC scaffold extracts or fresh $\alpha$-MEM medium for further assessment. After 1, 3 and 5 days of culture, the supernatant was replaced with $\alpha$-MEM medium containing $10 \%$ CCK8 solution (Dojindo Molecular Technologies Inc., Tokyo, Japan). After incubated at $37^{\circ} \mathrm{C}$ for $2 \mathrm{~h}$, the absorbance of supernatant was measured at $450 \mathrm{~nm}$.

\subsection{Release kinetics of VEGF in vitro}

Cylindrical HC-VEGF scaffolds $(n=3)$ were placed in $1 \mathrm{~mL}$ of PBS and incubated at $37^{\circ} \mathrm{C}$. At day $1,3,7,14,21$, and 28 , the supernatant was withdrawn and renewed with fresh PBS respectively. The collected supernatant was stored at $-80{ }^{\circ} \mathrm{C}$ until final test. Release kinetics of VEGF from HC-VEGF scaffolds was examined by using VEGF ELISA Kits (abcam, Cambridge, UK), according to the manufacturers' instructions.

\subsection{Surgical procedures}

Male Sprague-Dawley rats (SD rats: $250-300 \mathrm{~g}$ ) were used for animal experiments. All procedures were approved by the animal care committee of Peking Union Medical College Hospital. All experiments were performed in compliance with the Regulations for the Administration of Affairs Concerning Experimental Animals in China. SD rats were anesthetized with $10 \%$ chloral hydrate $(0.3 \mathrm{~mL} / 100 \mathrm{~g})$ by intraperitoneal injection and received unilateral operation to the left hindlimb. The hindlimb ischemia model was established by resecting femoral artery as described previously. ${ }^{\mathbf{9} 22,23}$ Briefly, a medial incision was made in the left thigh. The entire femoral artery from the inguinal ligament to saphenous/popliteal bifurcation was ligated, dissected and resected. HC (control group, $n=5$ ) or 


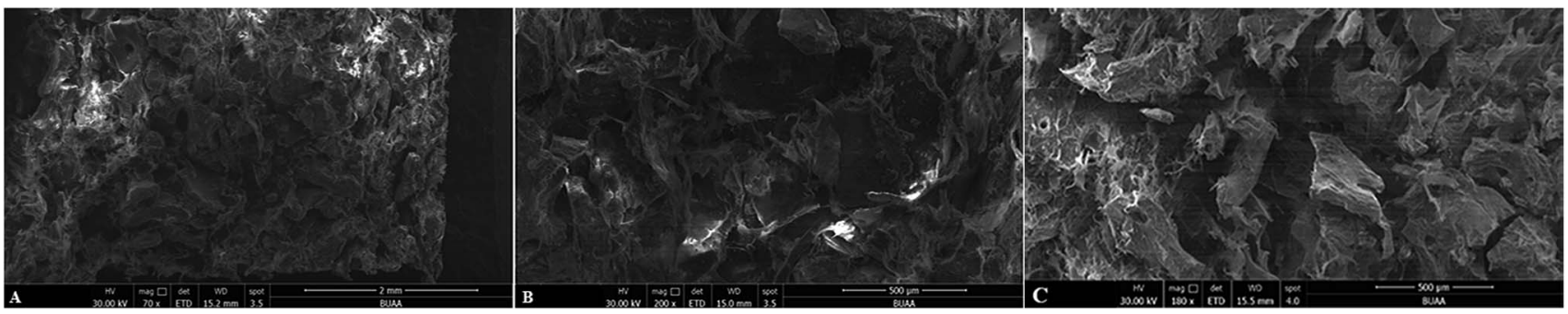

Fig. 1 SEM images of hydroxyapatite-collagen scaffold. Morphology and microstructures of HC scaffolds observed at different magnification (A/ B) and HC-VEGF scaffolds (C).

HC-VEGF (experiment group, $n=5$ ) scaffolds were implanted into intramuscular pockets created by blunt dissection in the left thigh. The wound was closed with 3-0 sutures. Animals were allowed to move freely after surgery.

\subsection{Samples harvest and preparation of tissue sections}

Implants were harvested at 8 weeks after implantation. The harvested samples were fixed in $4 \%$ formaldehyde solution, decalcified in EDTA, dehydrated in a graded series of ethanol, embedded in paraffin, and sectioned at $4 \mu \mathrm{m}$ of thickness.

\subsection{Histological analysis}

The sections were stained with hematoxylin and eosin (HE) and Masson's trichrome staining. The Masson's trichrome staining was used to identify the newly formed bone tissue. Three sections from each samples were selected for histomorphometric analysis. For each section, five regions of interest (ROI) including the center and the outer $1 / 3$ radius at $3,6,9$, and 12 o'clock $(100 \times$ magnification) were evaluated to calculate the percentage of bone area (bone area/total area). Quantification of new bone area was conducted by using Image $J$ software (National Institutes of Health, Bethesda). The mean percentage of bone area of each sample was calculated with all selected sections, which was then compared between HC scaffold and HC-VEGF scaffold groups.

\subsection{Immunohistochemical analysis}

Immunohistochemistry staining was conducted with antiCD31 antibodies to identify the microvessels in the samples as described previously. ${ }^{24}$ Expression of osteocalcin, a marker of bone formation, was also evaluated by immunohistochemistry staining after rupture of membranes and fixation of the sections. Firstly, endogenous peroxidase was blocked with $3 \%$ hydrogen peroxide before the treatment with $3 \%$ BSA for 30 min. After incubating with goat anti-mouse CD31 primary antibody (1:100, Wuhan Goodbio-technology Co., Ltd., Wuhan, China) or primary antibody against osteocalcin (Wuhan Goodbio-technology Co., Ltd., Wuhan, China) overnight at $4{ }^{\circ} \mathrm{C}$, slides were further incubated with secondary antibody (Dako, Denmark) for $50 \mathrm{~min}$. Then, 3,3'-diaminobenzidine was used as a chromogenic agent. Counterstaining was conducted with hematoxylin. Finally, the sections were dehydrated and coverslipped. The number of vessels in the above mentioned five ROI (200× magnification) was recorded with Image $\mathrm{J}$ software and presented as vessel density (vessels per $\mathrm{mm}^{2}$ ).

\subsection{Statistical analysis}

SPSS software (version 20.0, Chicago, Illinois, USA) was used for statistical analysis. Student's $t$-test was used to analyze the differences between groups. Statistically significant was defined as $p<0.05$. Data was expressed as mean \pm standard deviation (SD).

\section{Results and discussion}

\subsection{Characteristics and biocompatibility of HC scaffold}

Bone, as a natural composite material, mainly consists of collagen and hydroxyapatite. ${ }^{25}$ Apatite increases its strength and collagen keeps its toughness. HC scaffolds, similar to the natural bone in composition and microstructure, were widely investigated due to its biomimetic properties of bone tissue. ${ }^{25,26}$ The morphology and microstructures of HC scaffolds were evaluated by SEM. The scaffolds showed porous structures and intertwined assembly of fibrous microstructures (Fig. 1A and B), which was similar to the biomimetic hydroxyapatite-collagen scaffolds described in previous studies. ${ }^{20,25,26}$ The porous structures and fibrous microstructures are beneficial for cell and bone ingrowth. The addition of VEGF did not obviously change the morphology or microstructures of scaffolds (Fig. 1C). In the evaluation of

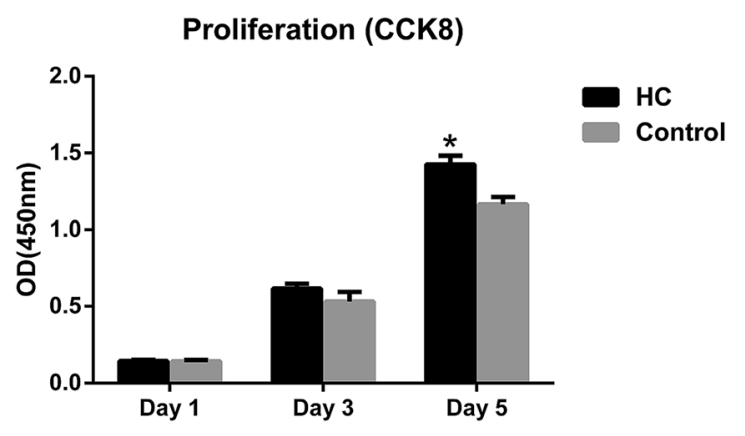

Fig. 2 Proliferation of MC3T3-E1 cells treated with HC scaffold extracts or $\alpha$-MEM medium. The proliferation of cells evaluated by CCK8 test was comparable between two tested groups after 1 and 3 days of culture. The OD value of cells treated with $\mathrm{HC}$ scaffold extracts was significantly higher than that in the control group after 5 days of culture $(P<0.05)$. 


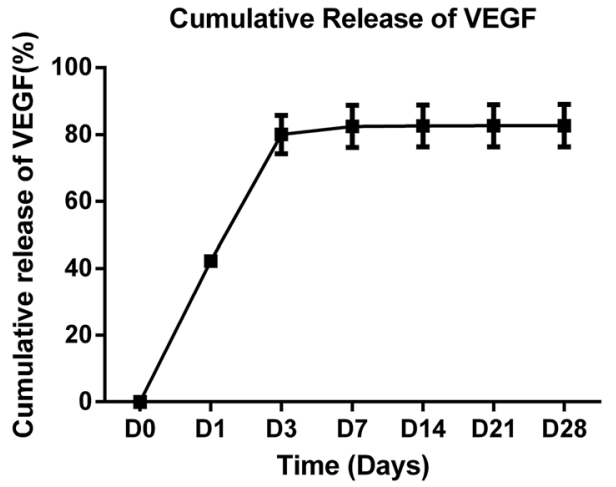

Fig. 3 Cumulative release of VEGF from HC-VEGF scaffolds $(n=3)$. An initial burst release ( $80 \%)$ of VEGF was detected during the first 3 days.

biocompatibility of HC scaffolds, CCK8 test was used to detect the viability of MC3T3-E1 cells treated with HC scaffold extracts or $\alpha$ MEM medium. The proliferation of cells was comparable between two tested groups after 1 and 3 days of culture. The OD value of cells treated with HC scaffold extracts was significantly higher than that in the control group after 5 days $(P<0.05)$ (Fig. 2). CCK8 assay indicated that the HC scaffolds had good cytocompatibility.

\subsection{Release kinetics of VEGF in vitro}

The effective delivery manner of VEGF in a composite scaffold was previously reported in the study of Kempen et al. ${ }^{27}$ Their results showed that the initial burst release of VEGF within the first 3 days was effective to enhance vascularization and bone formation induced by BMP-2. ${ }^{27}$ In this study, VEGF ELISA kit was used to quantify the VEGF released from HC-VEGF scaffolds $(n=3)$ at the selected time points. According to the procedure of scaffold fabrication, the total amount of VEGF added in each HC-VEGF scaffold was $1.33 \mu \mathrm{g}$. In vitro, the composite scaffolds also showed an initial burst release $(\sim 80 \%)$ of VEGF during the first 3 days, and the released VEGF was nearly undetectable after 7 days (Fig. 3). A total amount of $82.71 \%$ of VEGF was released in this observation period. The release of VEGF in the early stage can induce the establishment of local microcirculation, which is required for cell survival and bone regeneration.

\subsection{Angiogenesis and osteogenesis in an ischemic environment}

Blood supply plays a vital role in bone regeneration. ${ }^{7,28}$ It supplies oxygen, nutrients, growth factors and chemokines that is required for bone formation, and removes waste products that is detrimental to bone regeneration. ${ }^{7,29}$ Inhibition of
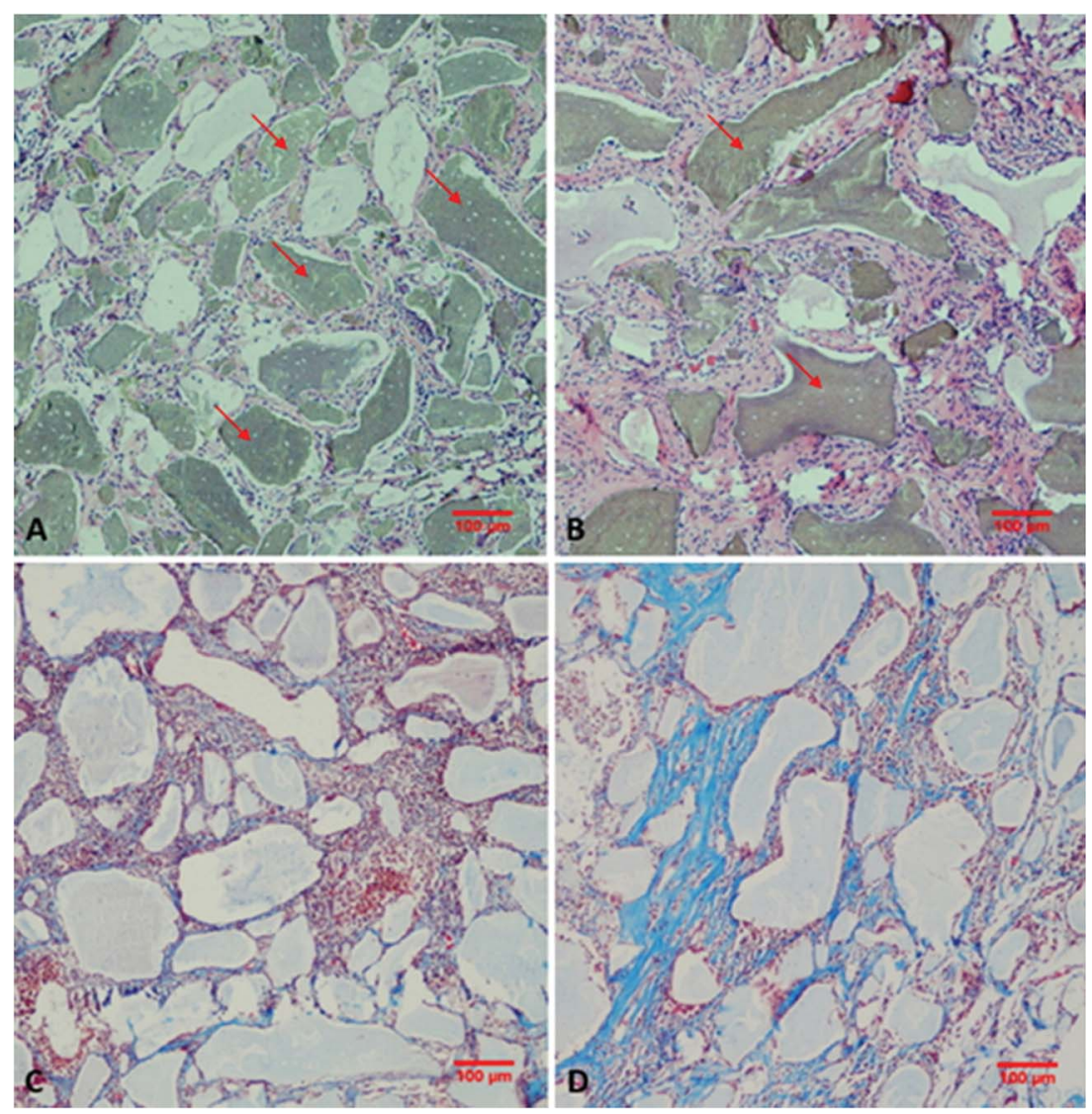

Fig. 4 Hematoxylin and eosin (A, B) and Masson's trichrome staining (C, D) of implanted HC scaffolds (A, C) and HC-VEGF scaffolds (B, D) (magnification $100 \times$ ). HE staining: HC-VEGF group (B) showed faster degradation and more tissue ingrowth than HC group (A) (grey area representative undegraded scaffolds, marked with red arrows). Masson's trichrome staining: bone formation in HC-VEGF group (D) was enhanced, compared to $\mathrm{HC}$ group (C) (blue area representative newly formed bone tissue). 
angiogenesis has been proven to be adverse to bone regeneration. ${ }^{30,31}$ Fang et $a l .{ }^{31}$ demonstrated that application of angiogenic inhibitor TNP-470 could block osteogenesis and result in fibrous nonunion. Similarly, the administration of VEGFspecific antagonist was also detrimental to angiogenesis and BMP4 induced bone regeneration. ${ }^{5}$ Improving blood supply has been demonstrated to be beneficial for bone formation. ${ }^{\mathbf{5} \mathbf{6}}$ For instance, transplantation of vascular bundle into bone substitute scaffold could enhance bone formation in ectopic and orthotopic sites. ${ }^{24,32-34}$ Unfortunately, some pathologic conditions, such as vascular injury, diabetes and arterial occlusion, may result in limb ischemia, ${ }^{9-11}$ which is a huge challenge for the successful treatment of bone fractures or defects in clinical. ${ }^{9,13}$ The incidence of impaired bone healing was reported to be as high as $46 \%$ in fractures accompanied with vascular injuries.9,13 That is significantly higher than the reported $10 \%$ non-union rate in general fractures. ${ }^{35-37}$ To investigate bone regeneration under ischemic environment, an ischemic model of femoral artery resection, which has been verified by many studies, ${ }^{9,12,21,22}$ was adopted in the present study. Blood supply and perfusion were significantly decreased after the resection of femoral artery. ${ }^{9,12,38} \mathrm{Lu}$ et $a{ }^{9}{ }^{9}$ showed that the resection of femoral artery severely reduced blood supply for the affected limb and created a persistent ischemic environment at the fracture site in a mouse model. Furthermore, the ischemic

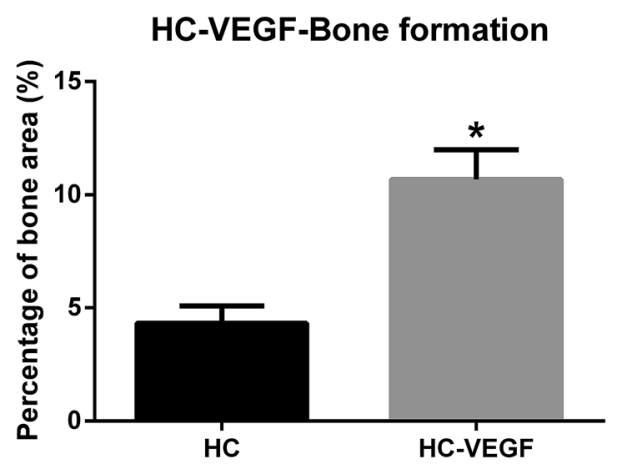

Fig. 5 Quantitative analysis of the percentage of bone area in $\mathrm{HC}$ and HC-VEGF groups $(n=5)$. The percentage of bone area in HC-VEGF group $(10.68 \%)$ was significantly increased than that in $\mathrm{HC}$ group $(4.32 \%)(P<0.05)$. insult finally resulted in a delayed or non-union in the fracture sites. ${ }^{9}$

A lots of strategies, such as growth factors delivery, stem cell transplantation, and vascular grafts, have been adopted to promote vascularized bone regeneration in bone tissue engineering. ${ }^{4,7,39}$ Among them, growth factors delivery is a classical and promising approach., ${ }^{4,7}$ VEGF, as a key mediator of angiogenesis, is one of the most investigated pro-angiogenic factors and has been proven to be able to improve vascularization and blood perfusion of skeletal muscle in the ischemic models. ${ }^{15,16}$ Tsurumi et al. ${ }^{16}$ reported that intramuscular injection of VEGF plasmid DNA could significantly increase the collateral vessels and capillaries in the treatment of acute limb ischemia. The expression of VEGF could also stimulate angiogenesis and improve blood supply in the ischemic lower limb secondary to diabetics. ${ }^{15}$ Improving blood perfusion may have the potential to enhance bone formation in an ischemic limb. For instance, the application of static magnetic field enhanced femoral bone formation due to improved blood supply in an ischemic rat model. ${ }^{21}$ The present study further investigated that whether VEGF-released from biomimetic HC scaffolds could improve angiogenesis and osteogenesis in an ischemic environment.

After resection of femoral artery of SD rats, HC and HC-VEGF scaffolds were implanted into intramuscular pockets created by blunt dissection in the left thigh. Eight weeks later, implants were retrieved for further assessment of bone formation by histological analysis and vascularization by immunohistochemical analysis. Results of HE staining showed faster degradation and more tissue ingrowth in HC-VEGF scaffolds than in HC scaffold (Fig. 4). According to Masson's trichrome staining and quantitative analysis results, the percentage of bone area in HC-VEGF group was significantly increased than that in HC group (10.68\% vs. $4.32 \%, n=5, P<0.05)$ (Fig. 5). In immunohistochemistry staining of bone formation marker, the expression of osteocalcin in HC-VEGF group was also much higher than that in HC group (Fig. 6), which further confirmed the results of Masson's trichrome staining. The effect of VEGF on angiogenesis in HC scaffolds was investigated by immunohistochemistry staining with anti-CD31 antibodies. The vessel sections were defined as a brown-yellow area with a recognizable lumen when observed by light microscopy (Fig. 7). The number of CD31 positive stained capillary vessels in HC-VEGF

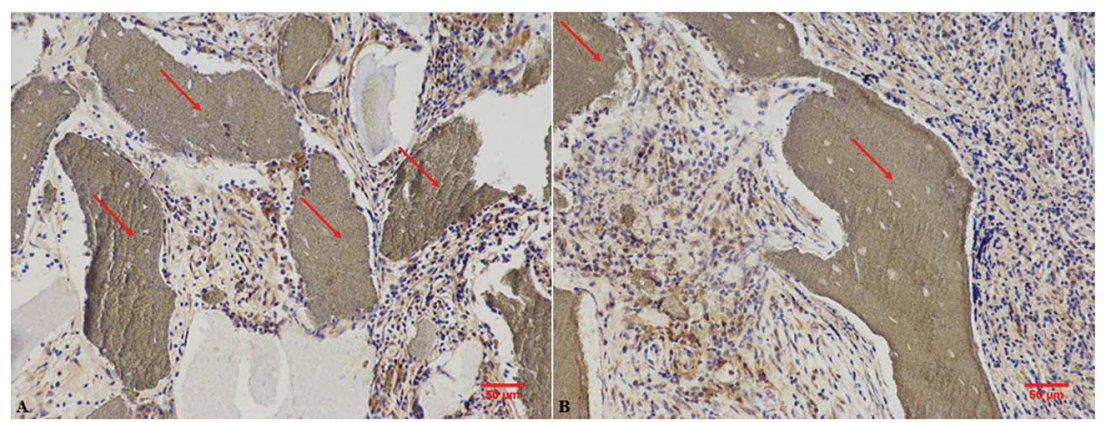

Fig. 6 Immunohistochemistry staining of bone formation marker. The expression of osteocalcin in HC-VEGF group (B) was much higher than that in $\mathrm{HC}$ group (A) (undegraded scaffolds marked with red arrows). 

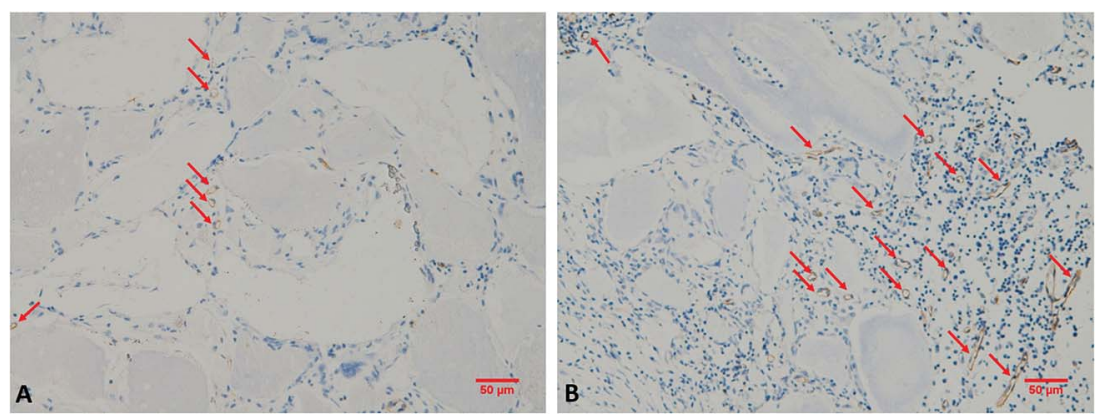

Fig. 7 Immunohistochemical analysis of angiogenesis. HC-VEGF group (B) showed more vascular invasion than HC group (A). The vessel sections were shown as a brown-yellow area with a recognizable lumen. Representative vessel sections were marked with red arrows.

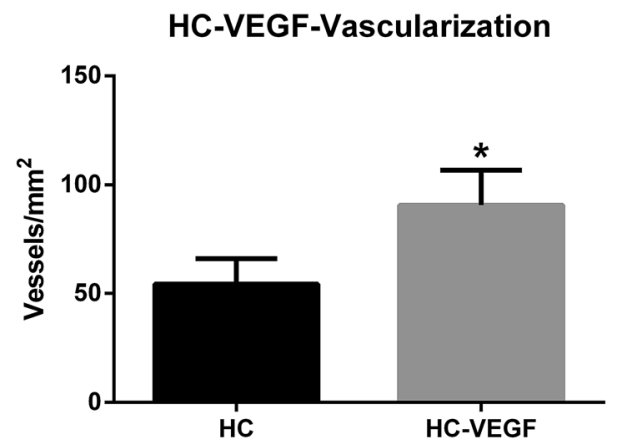

Fig. 8 Vessels density in HC and HC-VEGF scaffolds at 8 weeks after implanted. The numbers of CD31 positive stained capillary vessels in HC-VEGF group $\left(90.69 \mathrm{~mm}^{-2}\right)$ were significantly more than those in HC group $\left(54.30 \mathrm{~mm}^{-2}\right)(P<0.05)$.

group (90.69 $\mathrm{mm}^{-2}$ ) was significantly larger than that in $\mathrm{HC}$ group (54.30 $\mathrm{mm}^{-2}$ ) (Fig. 8). The release of VEGF significantly enhanced vascular invasion of HC scaffolds $(P<0.05)$. The results were consistent with the study of Kaigler et al. ${ }^{\mathbf{4 0}}$ which indicated that VEGF incorporated into PLGA scaffolds had the potential to increase vascularization and bone formation in an irradiated calvarial defect model. ${ }^{40}$

The relationship between vascularization and bone regeneration has been well-established. Enhanced neovascularization can be more effective to improve the supply of oxygen and nutrients and eliminate the waste products in the bone regeneration sites. In the present study, bone formation in HC-VEGF group was significantly enhanced compared to the HC group. The enhanced bone formation may be partly attribute to the improved angiogenesis in HC-VEGF scaffold. Besides, VEGF was reported to be able to directly and indirectly enhance bone formation..$^{27,41}$ It can increase vascular permeability and facilitate the recruitment of osteoprogenitor cells, which will indirectly enhance bone regeneration..$^{27,41}$ VEGF can also directly attract mesenchymal stem cells (MSCs) and promote their osteogenic differentiation. ${ }^{27,41}$ The accurate mechanisms need to be further investigated in the future studies.

\section{Conclusions}

The treatment of bone fractures and defects in an ischemic environment is a huge challenge in clinical. The investigation and understanding on this issue are insufficient. This study provided a very promising approach to enhance angiogenesis and osteogenesis in an ischemic environment by incorporating VEGF into biomimetic bone scaffolds.

\section{Conflicts of interest}

The authors disclose no potential conflict of interest.

\section{Acknowledgements}

This work was supported by the fundamental research funds for central public welfare research institutes, Chinese Academy of Medical Sciences (No. 2015PT320014), Peking Union Medical College graduate student innovation Fund (No. B2014001060), and the National High-Tech R\&D Program of China (863 Program, 2015AA020316, 2015AA033601).

\section{References}

1 A. E. Mercado-Pagan, A. M. Stahl, Y. Shanjani and Y. Yang, Ann. Biomed. Eng., 2015, 43, 718-729.

2 Y. Liu, J. K. Chan and S. H. Teoh, J. Tissue Eng. Regener. Med., 2015, 9, 85-105.

3 M. Lovett, K. Lee, A. Edwards and D. L. Kaplan, Tissue Eng., Part B, 2009, 15, 353-370.

4 L. H. Nguyen, N. Annabi, M. Nikkhah, H. Bae, L. Binan, S. Park, Y. Kang, Y. Yang and A. Khademhosseini, Tissue Eng., Part B, 2012, 18, 363-382.

5 H. Peng, A. Usas, A. Olshanski, A. M. Ho, B. Gearhart, G. M. Cooper and J. Huard, J. Bone Miner. Res., 2005, 20, 2017-2027.

6 E. Vogelin, N. F. Jones, J. I. Huang, J. H. Brekke and J. R. Lieberman, J. Bone Jt. Surg., Am. Vol., 2005, 87, 13231331.

7 U. Saran, S. Gemini Piperni and S. Chatterjee, Arch. Biochem. Biophys., 2014, 561, 109-117.

8 J. H. Holstein, M. Klein, P. Garcia, T. Histing, U. Culemann, A. Pizanis, M. W. Laschke, C. Scheuer, C. Meier, H. Schorr, T. Pohlemann and M. D. Menger, Br. J. Pharmacol., 2008, 154, 1055-1062. 
9 C. Lu, T. Miclau, D. Hu and R. S. Marcucio, J. Orthop. Res., 2007, 25, 51-61.

10 R. S. Smith Jr, L. Gao, L. Chao and J. Chao, Biol. Chem., 2008, 389, 725-730.

11 A. Reynolds, A. Sandstrom and P. K. Jha, Ann. Vasc. Surg., 2017, 38, e13-e16.

12 C. Lu, Z. Xing, Y. Y. Yu, C. Colnot, T. Miclau and R. S. Marcucio, J. Orthop. Res., 2010, 28, 687-696.

13 K. F. Dickson, S. Katzman and G. Paiement, Contemporary orthopaedics, 1995, 30, 489-493.

14 M. Sugano, K. Tsuchida and N. Makino, Circulation, 2004, 109, 797-802.

15 X. Li, K. Gan, G. Song and C. Wang, J. Diabetes. Complicat., 2015, 29, 872-881.

16 Y. Tsurumi, M. Kearney, D. Chen, M. Silver, S. Takeshita, J. Yang, J. F. Symes and J. M. Isner, Circulation, 1997, 96, Ii-382-388.

17 S. Almubarak, H. Nethercott, M. Freeberg, C. Beaudon, A. Jha, W. Jackson, R. Marcucio, T. Miclau, K. Healy and C. Bahney, Bone, 2016, 83, 197-209.

18 F. David, T. J. Levingstone, W. Schneeweiss, M. de Swarte, H. Jahns, J. P. Gleeson and F. J. O'Brien, J. Tissue Eng. Regener. Med., 2015, 9, 1193-1199.

19 J. P. Gleeson, N. A. Plunkett and F. J. O'Brien, European cells \& materials, 2010, 20, 218-230.

20 Z. Liu, X. Yin, Q. Ye, W. He, M. Ge, X. Zhou, J. Hu and S. Zou, J. Biomater. Appl., 2016, 31, 121-131.

21 S. Xu, N. Tomita, R. Ohata, Q. Yan and Y. Ikada, Bio-Med. Mater. Eng., 2001, 11, 257-263.

22 G. Tang, D. N. Charo, R. Wang, I. F. Charo and L. Messina, J. Vasc. Surg., 2004, 40, 786-795.

23 S. Takeshita, L. P. Zheng, E. Brogi, M. Kearney, L. Q. Pu, S. Bunting, N. Ferrara, J. F. Symes and J. M. Isner, J. Clin. Invest., 1994, 93, 662-670.

24 L. Wang, H. Fan, Z. Y. Zhang, A. J. Lou, G. X. Pei, S. Jiang, T. W. Mu, J. J. Qin, S. Y. Chen and D. Jin, Biomaterials, 2010, 31, 9452-9461.

25 S. Kuttappan, D. Mathew and M. B. Nair, Int. J. Biol. Macromol., 2016, 93, 1390-1401.
26 G. Calabrese, R. Giuffrida, C. Fabbi, E. Figallo, D. Lo Furno, R. Gulino, C. Colarossi, F. Fullone, R. Giuffrida, R. Parenti, L. Memeo and S. Forte, PLoS One, 2016, 11, e0151181.

27 D. H. Kempen, L. Lu, A. Heijink, T. E. Hefferan, L. B. Creemers, A. Maran, M. J. Yaszemski and W. J. Dhert, Biomaterials, 2009, 30, 2816-2825.

28 S. M. Chim, J. Tickner, S. T. Chow, V. Kuek, B. Guo, G. Zhang, V. Rosen, W. Erber and J. Xu, Cytokine Growth Factor Rev., 2013, 24, 297-310.

29 R. E. Unger, E. Dohle and C. J. Kirkpatrick, Adv. Drug Delivery Rev., 2015, 94, 116-125.

30 M. R. Hausman, M. B. Schaffler and R. J. Majeska, Bone, 2001, 29, 560-564.

31 T. D. Fang, A. Salim, W. Xia, R. P. Nacamuli, S. Guccione, H. M. Song, R. A. Carano, E. H. Filvaroff, M. D. Bednarski, A. J. Giaccia and M. T. Longaker, J. Bone Miner. Res., 2005, 20, 1114-1124.

32 H. Fan, X. Zeng, X. Wang, R. Zhu and G. Pei, Biomaterials, 2014, 35, 7407-7415.

33 S. Akita, N. Tamai, A. Myoui, M. Nishikawa, T. Kaito, K. Takaoka and H. Yoshikawa, Tissue Eng., 2004, 10, 789-795. 34 L. Cai, Q. Wang, C. Gu, J. Wu, J. Wang, N. Kang, J. Hu, F. Xie, L. Yan, X. Liu, Y. Cao and R. Xiao, Biomaterials, 2011, 32, 8497-8505.

35 Y. Liu, J. Lim and S. H. Teoh, Biotechnol. Adv., 2013, 31, 688705.

36 C. H. Lu, Y. H. Chang, S. Y. Lin, K. C. Li and Y. C. Hu, Biotechnol. Adv., 2013, 31, 1695-1706.

37 T. A. Einhorn, J. Bone Jt. Surg., Am. Vol., 1995, 77, 940-956.

38 C. Emanueli, A. Minasi, A. Zacheo, J. Chao, L. Chao, M. B. Salis, S. Straino, M. G. Tozzi, R. Smith, L. Gaspa, G. Bianchini, F. Stillo, M. C. Capogrossi and P. Madeddu, Circulation, 2001, 103, 125-132.

39 A. Hokugo, Y. Kubo, Y. Takahashi, A. Fukuda, K. Horiuchi, K. Mushimoto, S. Morita and Y. Tabata, Tissue Eng., 2004, 10, 978-986.

40 D. Kaigler, Z. Wang, K. Horger, D. J. Mooney and P. H. Krebsbach, J. Bone Miner. Res., 2006, 21, 735-744.

41 W. Zhang, X. Wang, S. Wang, J. Zhao, L. Xu, C. Zhu, D. Zeng, J. Chen, Z. Zhang, D. L. Kaplan and X. Jiang, Biomaterials, 2011, 32, 9415-9424. 